\title{
T-shaped spin filter with a ring resonator
}

\author{
A. A. Kiselevt and K. W. Kim \\ Department of Electrical and Computer Engineering, North Carolina State University, Raleigh, NC 27695-7911
}

\begin{abstract}
A planar ballistic structure is predicted to be highly effective in filtering electron spin from an unpolarized source into two output fluxes with the opposite and practically pure spin polarizations. The operability of the proposed device relies on the peculiar spin-dependent transmission properties of the T-shaped connector in the presence of the Rashba spin-orbit interaction as well as the difference in the dynamic phase gains of the two alternative paths around the ring resonator through upper and lower branches for even and odd eigenmodes.
\end{abstract}

PACS numbers: 73.63.-b; 72.25.-b

The high-vacuum experiments of early 1920s performed by Stern and Gerlach demonstrated that the trajectory of neutral silver atom is affected by the spin eigenvalue of its unpaired electron in the inhomogeneous magnetic field [1]. The observed effect is typically weak. With all recent attention to the spin related phenomena [2], the solid-state implementation of similar principles is of considerable interest. Propelled by the modern nanotechnology, the practically unlimited ability to modify the matter properties suggests a conceptual possibility to design a miniature spin filter of superior quality and effectiveness.

In this Letter we propose a planar three-terminal ballistic solid-state device that can filter an unpolarized incident electrons into two output channels according to the their spin orientation. The Stern-Gerlach nonuniform magnetic field is substituted by the intrinsic spinorbit (SO) interaction, naturally present in the lowsymmetry setups (for example, in semiconductor heterostructures) and acting on the electron spin as an effective momentum-dependent magnetic field. Various suggestions, how to utilize the $\mathrm{SO}$ effect, have recently been published in a number of spin-related proposals [3 6 . This approach is a potentially big advantage over the external sources of the strong magnetic field that are bulky and/or require extensive additional circuitry (though incorporation of the ferromagnetic films into the layered structure has also been considered [7]). We have analyzed several geometries, mainly symmetric and asymmetric $2 \mathrm{D}$ ballistic rings. Important property of this type of structures is the possibility of at least two alternative paths for the incoming electron that can lead to the interference and noticeable manifestations of the intrinsic SO effect in the spin-dependence of the transmission probabilities. The special geometry provides high effectiveness with up to $70 \%$ of the incident unpolarized flux filtered into two output fluxes with practically pure spin states.

The structure under consideration consists of an area of complex geometry formed from the two-dimensional electron gas using, e.g., an electrostatic split-gate technique, and connected to the exterior by a number of quasi-one dimensional (1D) wires. The simplest model 2D $(x-y$ plane) spin-independent electron Hamiltonian is complimented by the spin-related term (symmetrized to keep it Hermitian that is denoted here by curly brackets $\{\ldots\})$ :

$$
H=\hat{1} \frac{\hbar^{2}}{2 m}\left(k_{x}^{2}+k_{y}^{2}\right)+\left\{\eta(x, y)\left(\hat{\sigma}_{x} k_{y}-\hat{\sigma}_{y} k_{x}\right)\right\}
$$

Equation (11) defines the problem along with the boundary conditions on the two-component spinor wavefunction $\hat{u}=0$ at the structure edges (hard walls). The third dimension (i.e., the $z$ coordinate) is strongly quantized. The SO interaction can be caused, in particular, by the asymmetry in the $z$-confining potential (the socalled Rashba [8] or structure-asymmetry-induced term) with its strength given by the coefficient $\eta, \hat{\sigma}_{\alpha}$ are the Pauli matrices $(\alpha=x, y, z)$, and $\hat{1}$ is a $2 \times 2$ unity matrix. The $\eta$ in the first approximation is proportional to the $z$-component of the local electric field in the structure layer and depends on the properties of the semiconductor which forms 2D layer. For the practical means, this asymmetry in the confining potential can be formed and/or manipulated locally by the special control electrode(s) located over/under the structure [9]. For simplicity, we ignore other potential sources of SO interaction in our system (that nevertheless exist and compete in real systems) 10,11. We also leave aside any other phenomena beyond explicitly expressed in Eq. (1) (especially many-body interactions and all types of relaxation processes). We concentrate here exclusively on the stationary setup.

Transmission and reflection properties of the arbitrarily complex linear system connected to the exterior via $1 \mathrm{D}$ wires can be condensed into a finite-size scattering matrix $\mathbf{S}$ [12], consisting of the reflection coefficients $r_{i i}$ (in the $i$-th channel) and transmission coefficients $t_{i j}$ (describing propagation of the particle from the $j$-th into the $i$-th channel). We choose to enumerate as distinguishable channels (or terminals) all energetically allowed electron fluxes through different $1 \mathrm{D}$ subbands even of the same wire. Thus, the number of the channels can differ from the number of the attached leads. Having said that, we will be primarily interested in the situation when all connecting wires are identical and the incident electron en- 
ergy permits transmission solely through the ground subband. For example, in the hard-wall approximation the ground subband wavefunction in the ideal lead of width $w$ is a plane wave $\Psi^{ \pm}=\psi_{0}(y) \exp ( \pm i k x)$ with an envelope $\psi_{0}(y)=(2 / w)^{1 / 2} \cos (\pi y / w)$ that is symmetric in the perpendicular direction and corresponds to the quantization energy $E_{0}=\hbar^{2} \pi^{2} / 2 m w^{2}$ (these formulae are given in coordinates describing the channel along the axis $x$ ). The total energy of the state in the lead is $E=E_{0}+E_{\text {kin }}$ where $E_{\text {kin }}=\hbar^{2} k^{2} / 2 m$. For the kinetic energy interval $0<E_{\text {kin }}<3 E_{0}$ the propagation in the arms is possible only through the ground subbands.

In the case of a spinless particle, the $r_{i i}, t_{i j}$ are scalars. By taking electron spin into consideration, the number of transmission channels effectively doubles. If the spindependent interactions are not overly strong, and especially when they are spatially localized to the interior of the system (that is near the control electrodes in our case), it is convenient to just "upgrade" transmission and reflection coefficients of the $\mathbf{S}$ into $2 \times 2$ submatrices, thus keeping pairs of the channels differing only by the spin orientation together. Each $2 \times 2$ coefficient in this case can be conveniently expanded as

$$
\hat{x}=\hat{1} x_{1}+i \sum_{\alpha} \hat{\sigma}_{\alpha} x_{\alpha} \quad\left(x=r_{i i} \text { or } t_{i j}\right) .
$$

With the total flux $\mathbf{F} \propto \mathbf{U}^{+} \mathbf{U}$, the requirement of the flux conservation for $\mathbf{U}_{\text {out }}=\mathbf{S} \mathbf{U}_{\text {in }}$, corresponding to an arbitrary column $\mathbf{U}_{\text {in }}$ (symbolically representing coherent incident waves $\hat{u}_{i}$ coming through all channels), can only be secured if

$$
\mathbf{S}^{+} \mathbf{S}=\mathbf{1}
$$

We perform now the symmetry analysis of this system. For a broad class of problems including the one defined by Eq. (11), time-reversal invariance (with the operator $\hat{T}=$ $-i \hat{\sigma}_{y} K$ where $K$ is the complex conjugation) establishes the following relation on the scattering matrix $\mathbf{S}$

$$
\hat{\sigma}_{y} \mathbf{S}^{*} \hat{\sigma}_{y} \mathbf{S}=\mathbf{1}
$$

This equation should be regarded as a symbolic one, with the Pauli matrix multiplications applied to each submatrix $\hat{r}_{i i}, \hat{t}_{i j}$ separately.

Combined with Eq. (3), this relation can be converted into a more practical form $\hat{\sigma}_{y} \mathbf{S}^{*} \hat{\sigma}_{y}=\mathbf{S}^{+}$, that immediately results into

$$
r_{i i, \alpha}=0, t_{i j, 1}=t_{j i, 1}, t_{i j, \alpha}=-t_{j i, \alpha},
$$

Additional structure symmetry elements can provide further relations on the components of $\mathbf{S}$. For example, for the system symmetric in respect to the reflection $y \leftrightarrow$ $-y\left(S_{y}\right)$, the operator $-i \hat{\sigma}_{y} S_{y}$ commutes with the model Hamiltonian giving

$$
\hat{\sigma}_{y} S_{y}(\mathbf{S}) \hat{\sigma}_{y}=\mathbf{S}
$$

The effect of reflection $S_{y}$ on the scattering matrix is set by the permutation of the pairs of indices $(i, j, \ldots) \leftrightarrow$ $(k, l, \ldots)$ corresponding to the symmetrically located channels. In components, that leads to relations

$$
\begin{aligned}
r_{i i, 1}=r_{k k, 1}, t_{i j, 1} & =t_{k l, 1}, t_{i j, x}=-t_{k l, x} \\
t_{i j, y} & =t_{k l, y}, t_{i j, z}=-t_{k l, z}
\end{aligned}
$$

As a consequence, together with the relations of Eq. (5) this also suggests $t_{i k, y}=0$ for terminals $i \leftrightarrow k$. Another important case comes up when channels $i$ and $j$ are positioned along the reflection plane and as a result they reflect to themselves. For this setup $t_{i j, x}=t_{i j, z}=0$.

Similar relations take place for the reflection $x \leftrightarrow-x$ $\left(S_{x}\right)$. As for the $z \leftrightarrow-z\left(S_{z}\right)$, it changes the sign of the intrinsic electric field responsible for the Rashba SO interaction, thus establishing relations between scattering matrices $\mathbf{S}(\eta)$ and $\mathbf{S}(-\eta)$ as

$$
\hat{\sigma}_{z} \mathbf{S}(-\eta) \hat{\sigma}_{z}=\mathbf{S}(\eta)
$$

These simple qualitative considerations suffice for the purposes of the present Letter 13].

An elementary channel flux $F \propto \hat{u}^{+} \hat{u}$ with the $100 \%$ polarization is conveniently given by the spinor column $\hat{u}=\left(u_{\uparrow}, u_{\downarrow}\right)$. The relative magnitude and phase between $u_{\uparrow}$ and $u_{\downarrow}$ characterize orientation of the spin; vector $\boldsymbol{P}$ defined by three components $P_{\alpha}$ in the $x y z$ coordinate system is called the polarization vector, $P_{\alpha} F \propto \hat{u}^{+} \hat{\sigma}_{\alpha} \hat{u}$. For the arbitrary spinor $\hat{u}$ the absolute value $|\boldsymbol{P}|=1$. Being pumped into the channel $j$, the $\hat{t}_{i j} \hat{u}$ part of the incident flux will seep through the structure into the $i$ output channel. In the general case of spin-dependent interactions present in the system, $\hat{t}_{i j, \alpha} \neq 0$ and the spin will rotate from its original orientation. Moreover, the magnitude of the transmitted flux will depend on the spin orientation of the incident flux.

Partially polarized electron fluxes can be mimicked by a number of independent (not phase coherent) elementary fluxes $\hat{u}_{q}$ through the same channel that are just additive in the case of our linear system

$$
F=\sum_{q} F_{q}, \boldsymbol{P F}=\sum_{q} \boldsymbol{P}_{q} F_{q} .
$$

To present the unpolarized input flux in channel $j$, we use, for example, two elementary fluxes $\hat{u}_{1}=(1,0)$ and $\hat{u}_{2}=(0,1)$. Now it is very easy to evaluate $F, \boldsymbol{P}$ for the electron flux, transmitted into channel $i$. Indeed,

$$
F \propto\left|t_{i j, 1}\right|^{2}+\left|t_{i j, x}\right|^{2}+\left|t_{i j, y}\right|^{2}+\left|t_{i j, z}\right|^{2}
$$

with the $x$-component of spin polarization

$$
P_{x} F \propto 2 \operatorname{Im}\left(t_{i j, 1} t_{i j, x}^{*}+t_{i j, y}^{*} t_{i j, z}\right)
$$


and $P_{y}, P_{z}$ obtained by the cyclic permutation of indices. This equation shows, in particular, that if only one component of $\hat{t}_{i j}$ is present, polarization of the transmitted electron flux is zero for unpolarized input.

With the polarization vector $\boldsymbol{P}$ of the transfered flux expressed that way, we can come to another very important consequence with the help of Eqs. (3) and (4): the system with the Hamiltonian of Eq. (1) and just two connecting terminals cannot polarize transmitted electron flux. This conclusion is generally relaxed for the structures with three or more terminals (see also 14 in relation to this matter). We restrict ourselves here to three-terminal devices.

Quickly, we recollect now the results [15], obtained for the simple T-shaped structure, formed by the confining potential of two intersecting 1D channels [see Fig. 11(a)]. The SO interaction is formed in the $2 \mathrm{D}$ electron gas layer by gate electrodes and localized to the intersection area only. As the unpolarized electrons enter the input arm $(-x)$, the quantities of interest are the magnitudes and polarizations of the two output fluxes through the $\pm y$ arms. With the $S_{y}$-symmetric T-shaped structure providing symmetry relations of Eqs. (6) and (7), the total transmitted fluxes of the two output channels are the same but their polarizations along the $x$ and $z$ axes are opposite $\left(P_{y}\right.$ is the same). The transmission varies vary gradually with the incident energy but has low dips in the vicinity of the resonances with the quasi-localized 0D electron levels at the intersection (corresponding to the total reflection in the case of no SO). The SO interaction forms also a fine structure near these resonances. In the resonance regions where the difference between the incident energy and the energy of the quasi-localized 0D state becomes comparable to the SO term, high values of polarization take place. Actually, the smaller the SO term is, the narrower these regions of high polarization and the higher (closer to 100\%) the polarization values become. Note, though, that this happens at the expense of diminishing total transmission.

The same T-shaped spin filter with an attached ring resonator is shown in Fig. 11(b). In Figure 2, we present the energy dependence of the total transmitted flux $F$ (light area) and its polarized part $|\boldsymbol{P}| F$ (dark) into one of the two symmetric output channels (the flux conservation ensures that $F \leq 0.5)$. The data shown are obtained for $\eta=0$ (no SO), $\eta_{0}$ (relatively small $\mathrm{SO}$ ), and $3 \eta_{0}$ (stronger $\mathrm{SO})$ that are given in graphs (a)-(c), respectively. Coefficient $\eta_{0}=6 \mu \mathrm{eV} \cdot \mu \mathrm{m}$ is a reasonable basic value for the Rashba constant in InAs/InGaAs heterostructures [16], electron effective mass $m=0.023 m_{0}$ where $m_{0}$ is the free electron mass, the channel width $w=0.1 \mu \mathrm{m}$, and the ring radius $R=0.2 \mu \mathrm{m}$ [dashed circle in Fig. 11(b)]. This set of parameters gives the ground subband quantization energy $E_{0}=1.6 \mathrm{meV}$; thus, $3 \eta_{0}$ is equal to $0.11 \times E_{0} w$. We (arbitrarily) assume the SO coefficient $\eta(r)$ to have the spatial dependence $\eta(r)=\eta /\left[1+e^{\left(r-r_{0}\right) / \Delta r}\right]$, where $\eta$ and $r_{0}=0.1 \mu \mathrm{m}$ are defined by the back and front electrode potentials and their sizes, $\Delta r=0.025 \mu \mathrm{m}$ is included to account qualitatively for the fringe fields, $r=\left[(x+R)^{2}+y^{2}\right]^{1 / 2}$. For a numerical solution, we have applied the recursive method of Usuki et al. [17.

In the absence of the SO interaction [Fig. 2(a)], only $t_{i j, 1}$ terms are present in the transmission coefficients and the polarization of the output fluxes repeats that of the incident fluxes; thus, it is zero for the unpolarized input flux. Energy dependence of the transmission probability consists of a number of resonant peaks, corresponding to the eigenstates in the ring. Strictly speaking, eigenstates in the ideal ring should be defined via the Bessel functions. Under the assumption $w \ll 2 \pi R$, energies of several first states can be approximated by $E_{R, l}=k_{l}^{2} / 2 m$ (counted from the quantization energy $E_{0}$ in the ideal channel of width $w$ ), where $k_{l}=l / R$ and $l$ is an integer. Thus, except for the ground state $l=0$, the eigenstates in the ring are quadruply degenerate - twice because of the orbital motion $\pm l$, and twice due to the spin. Presence of the attached terminal leads inflicts a finite lifetime of the ring eigenmodes (and leads to some renormalization of the energies).

The arrows in the upper part of the graph indicate several first energies $E_{r, l}$. One can immediately note that for even orbital numbers $l$, transmission peaks are well defined, while for odd $l$, the peaks are suppressed and the main feature here is a dip in the transmission probability. This can qualitatively be explained as follows. An electron wave transferring to, say, upper output channel passes $1 / 4$ of the ring if moving through the upper branch $A \rightarrow C$ and $3 / 4$ for the lower branch $A \rightarrow B \rightarrow C$. Thus, the difference in the gained dynamic phase [18] is $\pi l$ that gives a multiplier of +1 for even and -1 for odd modes that secures either constructive or destructive interference at the output connector. Deviation from the described scenario, especially for a couple of the first eigenmodes can be explained by the leakage of the electron wave in the lower branch into the $-y$ lead (the leakage is stronger for smaller $E_{\text {kin }}$ ), thus, severely disturbing the balance of the wave amplitudes arriving via two paths.

Now it is easy to understand the structure of the transmission spectra in the presence of the SO interaction [Figs. 2(b) and 2(c)]. All peaks are split into two subpeaks because the four-fold degeneracy of the eigenstates in the ring is lifted into two pairs by the SO mixing [19]. Taking into consideration the symmetry relations of Eqs. (6) and (7) for the $S_{y}$-symmetric T-shaped connector, one can conclude that the explanation given above is still valid for the $t_{i j, 1}, t_{i j, y}$ components of the transferred flux, but for the $t_{i j, x}$ and $t_{i j, z}$, where the sign for the waves transmitted into the upper and lower branches are opposite, constructive interference takes place for odd eigenmodes in the ring. That is indeed very important conclusion since constructive interference of the $x-z$ polarized and unpolarized electron fluxes take place for 
drastically different incident energies. This explains really high polarization for the energies corresponding to the odd modes - the polarization reaches $100 \%$ where the dark-area $(|\boldsymbol{P}| F)$ and the light-area $(F)$ contour boarders adjoin.

Another important observation concerns the $P_{y^{-}}$ component of the transmitted flux. It is the same for both $+y$ and $-y$ output fluxes in the symmetric structure. That means that for the odd eigenmodes the corresponding flux component interferes destructively, just like the unpolarized flux fracture. Thus, $P_{y} \approx 0$ and polarizations of two output fluxes are not only extremely high, but they are also practically exactly opposite for two output arms, which is indeed an optimal situation for the filter.

So far we have implicitly considered monoenergetic incident electrons. For the described effect to be observable in a more realistic setup, the energy distribution of the incident electrons should not substantially exceed the energy gap between adjacent ring eigenmodes and be aligned in such a way that to overlap primarily with only one subpeak of the SO split transmission peak, as the spin polarization is approximately opposite for two subpeaks. These requirements could be fulfilled by adjusting the Fermi levels of contact reservoirs and performing experiments in a proper temperature range.

In summary, we have proposed a device consisting of the T-shaped spin filter with an attached ring resonator. Matching incident electron energies to some of the eigenmodes in the ring, we have been able to achieve both a superior spin selectivity and high transmission efficiency.

This work was supported by the ONR and DARPA.

* kiselev@eos.ncsu.edu

[1] W. Gerlach and O. Stern, Z. f. Phys. 9, 349 (1922);

[2] G. A. Prinz, Physics Today 48(4), 58 (1995); Science 282, 1660 (1998); D. J. Monsma et al., Phys. Rev. Lett. 74, 5260 (1995); J.-Ph. Ansermet, J. Phys.: Condens. Matter 10, 6027 (1998); B. E. Kane, Nature 393, 133 (1998); R. Vrijen and E. Yablonovitch, Physica E 10, 569 (2001).

[3] S. Datta and B. Das, Appl. Phys. Lett. 56, 665 (1990).

[4] A. G. Aronov and Yu. B. Lyanda-Geller, Phys. Rev. Lett. 70, 343 (1993).

[5] E. N. Bulgakov et al., Phys. Rev. Lett. 83, 376 (1999).

6] M. Governale, D. Boese, U. Zülicke, and C. Schroll, condmatt/0108373 (2001).

[7] C. Cacho, Y. Lassailly, H.-J. Drouhin, G. Lampel, and J. Peretti, Phys. Rev. Lett. 88, 066601 (2002).

[8] E. I. Rashba, Solid State Phys. 2, 1109 (1960).

[9] D. Grundler, Phys. Rev. Lett. 84, 6074 (2000).

[10] G. Lommer, F. Malcher, and U. Rössler, Phys. Rev. Lett. 60, 728 (1988).

[11] O. Krebs and P. Voisin, Phys.Rev. Lett. 77, 1829 (1996).

[12] S. Datta, Electronic transport in mesoscopic systems, (Cambridge University press, Cambridge, 1997), Ch. 3.

[13] Some problems require a quantitative analysis of the spin-dependent terms in the connector scattering matrix; A. A. Kiselev and Y. B. Lyanda-Geller, in preparation.

[14] P. Bruno, Phys. Rev. Lett. 79, 4593 (1997); T. P. Pareek and P. Bruno, Phys. Rev. B 63, 165424 (2001); U. Zulicke and C. Schroll, Phys. Rev. Lett. 88, 029701 (2002).

[15] A. A. Kiselev, K. W. Kim, Appl. Phys. Lett. 78, 775 (2001).

[16] Yu. B. Lyanda-Geller, Phys. Rev. Lett. 71, 657 (1993).

[17] T. Usuki et al., Phys. Rev. B 52, 8244 (1995).

[18] Due to the localization of the SO interaction in a small area at the left connector, topological phase of 4 is not involved here.

[19] For the particular case depicted in Fig. 1(b) with the Rashba SO interaction localized at the left connector, these eigenstate pairs are: $(l, 1 / 2)$ (the orbital number $l$ and the spin oriented along axis $x$ with $\left.s_{x}=1 / 2\right)$ and $(-l,-1 / 2)$; the second pair is $(l,-1 / 2)$ and $(-l, 1 / 2)$.

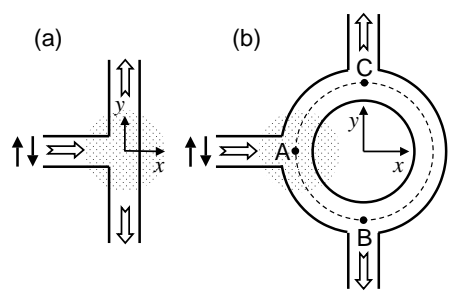

FIG. 1. (a) T-shaped spin filter with SO interaction induced by the control electrode(s) placed at the intersection; (b) the same structure with an attached ring resonator.

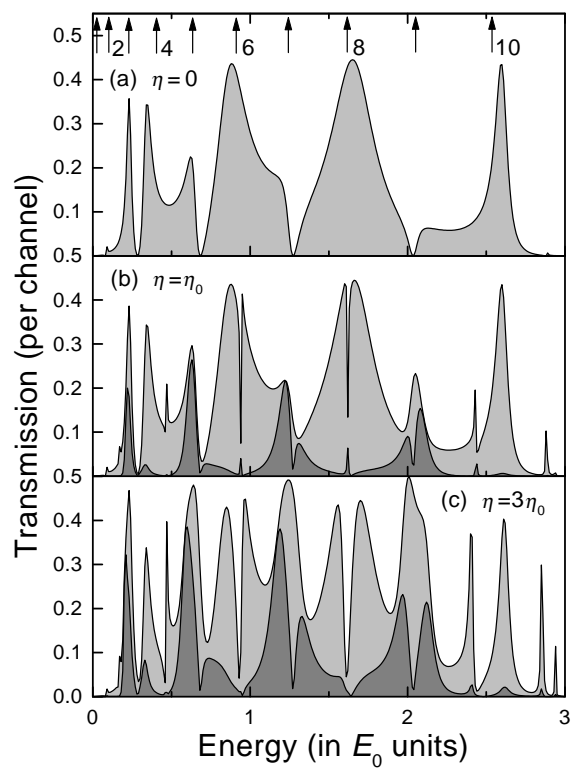

FIG. 2. Transmission of incident electron flux into output channel of the symmetric T-shape structure with a ring resonator as a function of electron kinetic energy. (a) No SO interaction at the input T-shaped connector, $\eta=0$; (b) $\eta=\eta_{0} ;$ (c) $\eta=3 \eta_{0}$ (stronger SO interaction). The light area represents total transmitted flux and the dark area shows its polarized part. The numbered arrows at the top denote approximate eigenmode energies $E_{R, l}$ in the ring. 